\title{
Exponential Stability of Switched Positive Homogeneous Systems
}

\author{
Dadong Tian and Shutang Liu \\ College of Control Science and Engineering, Shandong University, Jinan 250061, China \\ Correspondence should be addressed to Shutang Liu; stliu618@163.com
}

Received 9 March 2017; Revised 19 September 2017; Accepted 25 October 2017; Published 21 November 2017

Academic Editor: Pietro De Lellis

Copyright (C) 2017 Dadong Tian and Shutang Liu. This is an open access article distributed under the Creative Commons Attribution License, which permits unrestricted use, distribution, and reproduction in any medium, provided the original work is properly cited.

\begin{abstract}
This paper studies the exponential stability of switched positive nonlinear systems defined by cooperative and homogeneous vector fields. In order to capture the decay rate of such systems, we first consider the subsystems. A sufficient condition for exponential stability of subsystems with time-varying delays is derived. In particular, for the corresponding delay-free systems, we prove that this sufficient condition is also necessary. Then, we present a sufficient condition of exponential stability under minimum dwell time switching for the switched positive nonlinear systems. Some results in the previous literature are extended. Finally, a numerical example is given to demonstrate the effectiveness of the obtained results.
\end{abstract}

\section{Introduction}

Positive systems are those systems whose state variables and output signals are always contained in the first quadrant whenever both the initial conditions and input signals are nonnegative. Many real-word processes are described by positive systems. Examples of this type can be found in areas such as ecology, biology, and chemical engineering [1-3]. Due to their importance and wide applications, there has been an increasing interest in such systems.

Many important and interesting properties of positive systems have been reported and analyzed. It is well known that the most fundamental one is the stability property. Up until now the stability analysis of positive linear timeinvariant (LTI) systems is well developed $[4,5]$. In [4], it was shown that the delayed positive linear system $\dot{\mathbf{x}}(t)=$ $A \mathbf{x}(t)+B \mathbf{x}(t-\tau)$ was globally asymptotically stable (GAS) for all $\tau \geq 0$ if the corresponding delay-free system $\dot{x}(t)=(A+$ $B) \mathbf{x}(t)$ was GAS. This implies that such system is insensitive to certain class of time delays. Moreover, the exponential stability of positive linear systems with constant time delays was investigated by Zhu et al. [5], in which it was also shown that the decay rate depended on the magnitude of delays. Recently, some progress on the positive nonlinear systems called cooperative homogeneous systems has been made
[6-9]. For example, [6] derived a necessary and sufficient condition for exponential stability of such positive systems with the homogeneity of a degree of one.

On the other hand, switched systems have attracted much research attention in control theory field [10-13]. Typically, a switched system is a type of hybrid dynamical system consisting of family (either discrete-time or continuous-time) subsystems and a rule that regulates the switching among them. It has been widely applied in many areas, such as chemical processing and traffic control. For such systems, the stability theory is also one of the fundamental problems. The authors in [12] applied the Lyapunov-based theory, such as the multiple Lyapunov function, to study the stability problems.

Furthermore, switched positive systems combining the features of positive systems and switched systems are recently studied [14-18]. As switched positive systems are defined on cones rather than on linear spaces, studying such systems is more challenging than that of general switched systems. In recent years, some results concerning the switched positive systems have been reported. For example, the stability problem of switched positive linear systems has been extensively discussed by the approach of linear copositive functions such as $[14,15,17]$. In [19], the author showed a necessary and 
sufficient condition for exponential stability of a class of switched positive nonlinear systems under average dwell time switching.

It is worth noting that the results in [7] only dealt with asymptotical stability of positive systems. As is known to us, switched systems may be unstable even if we ensure that all the subsystems are stable. Therefore, it is meaningful to combine the positive systems with switched systems. On the other hand, the author of [19] only studied the switched positive systems with the homogeneity of a degree of one, in which the common exponential decay rate for all subsystems independent of initial conditions can be easily found for all subsystems. However, when the degree is not constrained to be one, the exponential decay rate depends on initial conditions, which would make the problem be more complicated. The state at each switched instant should be considered. In this case, it is worth studying whether it is possible to design suitable switching signals to keep the system exponentially stable. In this paper, by using the approach in which the Lyapunov-Krasovskii functional method is not included, we design appropriate time-dependent switching rules under which the switched positive nonlinear system is exponentially stable with the degree $0<\alpha \leq 1$.

The main contributions are summarized as follows. We derive a sufficient condition for exponentially stable unswitched positive systems of degree of $0<\alpha \leq 1$ with bounded time delays. It is shown that the decay rate depends on the initial conditions and the time delays. In particular, for the corresponding delay-free systems, the sufficient condition is also necessary. Then as special switched positive nonlinear systems, we consider the switched positive homogeneous systems with the degree of $0<\alpha \leq 1$. A sufficient condition for the exponential stability of switched positive homogeneous systems under minimum dwell time (MDT) switching is presented.

The layout of the paper is as follows. In Section 2, we introduce the notation and review some preliminaries. The main results of this paper are stated in Section 3. Section 4 provides a numerical example to show the validity of our results. Finally, concluding remarks are given in Section 5.

\section{Notation and Preliminaries}

2.1. Notation. Throughout the paper, vectors are written in bold lowercase letters. Let $\mathbb{R}, \mathbb{N}$, and $\mathbb{N}_{0}$ denote the set of real numbers, natural numbers, and the set of natural numbers including zero, respectively. $\mathbb{R}^{n}$ stands for the $n$-dimensional Euclidean space. Let $\mathbb{R}_{+}^{n}$ be the set of all vectors in $\mathbb{R}^{n}$ with nonnegative entries; that is, $\mathbb{R}_{+}^{n}:=\left\{\mathbf{x} \in \mathbb{R}^{n}, x_{j} \geq 0,1 \leq j \leq n\right\}$. For two vectors, $\mathbf{x}, \mathbf{y} \in \mathbb{R}^{n}$, we write $\mathbf{x} \geq \mathbf{y}$, if $x_{j} \geq y_{j}$, for $1 \leq$ $j \leq n ; \mathbf{x}>\mathbf{y}$, if $\mathbf{x} \geq \mathbf{y}$ and $\mathbf{x} \neq \mathbf{y} ; \mathbf{x} \gg \mathbf{y}$, if $x_{j}>y_{j} 1 \leq j \leq n$. $x_{j}$ is used to denote the $j$ th component of $\mathbf{x}$. Similarly, $x_{p j}$ denotes the $j$ th coordinate of vector $\mathbf{x}_{\mathbf{p}}$. In [7], given a vector $v \gg \mathbf{0}$, the weighted $l_{\infty}$ norm is defined by

$$
\|\mathbf{x}\|_{\infty}^{v}=\max _{1 \leq j \leq n} \frac{\left|x_{j}\right|}{v_{j}} .
$$

For matrix $A \in \mathbb{R}^{n \times n}$, let $a_{i j}$ denote its entry in row $i$ and column $j$. A real $n \times n$ matrix $A=\left(a_{i j}\right)_{n \times n}$ is Metzler if and only if its off-diagonal entries $a_{i j}, i \neq j$ are nonnegative.

For a real interval $[a, b]$, let $C\left([a, b], \mathbb{R}^{n}\right)$ be the space of all continuous functions on $[a, b]$ taking values in $\mathbb{R}^{n}$. The upper-right Dini derivative of a continuous function $h: \mathbb{R} \rightarrow$ $\mathbb{R}$ is denoted by $D^{+} h(\cdot)$.

$$
\left.D^{+} h(t)\right|_{t=t_{0}}=\lim _{\Delta \rightarrow 0^{+}} \sup \frac{h\left(t_{0}+\Delta\right)-h\left(t_{0}\right)}{\Delta} .
$$

2.2. Preliminaries. Next, we present some definitions and results which are used in this paper.

Definition 1. A vector field $\mathbf{f}: \mathbb{R}^{n} \rightarrow \mathbb{R}^{n}$ is called homogeneous of degree $\alpha>0$, if, for all $\mathbf{x} \in \mathbb{R}^{n}$ and $\lambda \in \mathbb{R}$, $\mathbf{f}(\lambda \mathbf{x})=\lambda^{\alpha} \mathbf{f}(\mathbf{x})$.

Definition 2. A continuous vector field $\mathbf{f}: \mathbb{R}^{n} \rightarrow \mathbb{R}^{n}$ which is continuously differentiable on $\mathbb{R}^{n} \backslash\{\mathbf{0}\}$ is said to be cooperative if the Jacobian matrix $(\partial \mathbf{f} / \partial \mathbf{x})(\mathbf{a})$ is Metzler for all $\mathbf{a} \in \mathbb{R}_{+}^{n} \backslash\{\mathbf{0}\}$.

It follows from [1, Remark 3.1] that the cooperative systems satisfy the following property.

Proposition 3. Let $\mathbf{f}: \mathbb{R}^{n} \rightarrow \mathbb{R}^{n}$ be cooperative. For any two vectors $\mathbf{x}$ and $\mathbf{y}$ in $\mathbb{R}_{+}^{n}$ with $\mathbf{x} \geq \mathbf{y}$ and $x_{j}=y_{j}$, one has $f_{j}(\mathbf{x}) \geq$ $f_{j}(\mathbf{y})$.

Definition 4. A vector field $\mathbf{g}: \mathbb{R}^{n} \rightarrow \mathbb{R}^{n}$ is said to be orderpreserving on $\mathbb{R}_{+}^{n} \backslash\{\mathbf{0}\}$, if $\mathbf{g}(\mathbf{x}) \geq \mathbf{g}(\mathbf{y})$ for any $\mathbf{x}, \mathbf{y} \in \mathbb{R}_{+}^{n} \backslash\{\mathbf{0}\}$ such that $\mathbf{x} \geq \mathbf{y}$.

\section{Main Results}

3.1. Exponential Stability of Unswitched Positive Homogeneous Systems. We first consider positive system with time-varying delays:

$$
\begin{aligned}
& \dot{\mathbf{x}}(t)=\mathbf{f}(\mathbf{x}(t))+\mathbf{g}(\mathbf{x}(t-\tau(t))), \quad t \geq 0, \\
& \mathbf{x}(t)=\boldsymbol{\theta}(t), \quad t \in\left[-d_{\text {max }}, 0\right]
\end{aligned}
$$

where $\mathbf{x}(t) \in \mathbb{R}^{n}$ is the state variable and $\mathbf{f}, \mathbf{g}: \mathbb{R}^{n} \rightarrow \mathbb{R}^{n}$ are continuous vector fields on $\mathbb{R}^{n}$, continuously differentiable on $\mathbb{R}^{n} \backslash\{\mathbf{0}\} . \boldsymbol{\theta}(t) \in C\left(\left[-d_{\max }, 0\right], \mathbb{R}^{n}\right)$ is the initial condition. In addition, we assume that system (3) satisfies the following conditions.

Assumption 5.

(i) $\mathbf{f}$ and $\mathbf{g}$ are homogeneous of degree of $\alpha>0$.

(ii) $\mathbf{f}$ is cooperative and $\mathbf{g}$ is order-preserving on $\mathbb{R}_{+}^{n} \backslash\{\mathbf{0}\}$.

Assumption 6. The time-varying delay $\tau(t): \mathbb{R}_{+} \rightarrow \mathbb{R}_{+}$is continuous and satisfies

$$
0 \leq \tau(t) \leq d_{\max }
$$

Here $\tau(t)$ is not necessarily continuously differentiable and no restriction on its derivative such as $\dot{\tau}(t)<1$ is imposed. 
Remark 7. Condition (i) of Assumption 5 implies that $\mathbf{f}(\mathbf{0})=$ $\mathbf{g}(\mathbf{0})=\mathbf{0}$. Furthermore, as $\mathbf{f}$ is cooperative and $\mathbf{g}$ is orderpreserving on $\mathbb{R}_{+}^{n}$, one has

$$
\begin{aligned}
& x_{j}=0 \Longrightarrow \\
& f_{j}(\mathbf{x}) \geq 0, \\
& \forall j \in\{1,2, \ldots, n\}, \forall \mathbf{x} \in \mathbb{R}_{+}^{n} \\
& \mathbf{g}(\mathbf{x}) \geq \mathbf{0}, \quad \forall \mathbf{x} \in \mathbb{R}_{+}^{n} .
\end{aligned}
$$

It has been shown in [7, Proposition 3.2] that (5) implies that system (3) is positive for any initial condition $\boldsymbol{\theta}(\cdot) \in$ $\mathbb{R}_{+}^{n}$. Throughout this paper, we always assume that the initial conditions are nonnegative.

Before giving the sufficient condition for the exponential stability of system (3), a lemma that provides a precise estimation of the upper bound of system (3) is proposed first.

Lemma 8 (see [7]). Consider system (3) under Assumptions 5 and 6. If there exists a vector $\boldsymbol{v} \gg \mathbf{0}$ such that $\mathbf{f}(\boldsymbol{v})+\mathbf{g}(\boldsymbol{v}) \ll \mathbf{0}$, then the solution $\mathbf{x}(t)$ satisfies

$$
\|\mathbf{x}(t)\|_{\infty}^{\boldsymbol{v}} \leq\|\boldsymbol{\theta}\|, \quad t \in[0,+\infty),
$$

where $\|\boldsymbol{\theta}\|=\sup _{-d_{\max } \leq t \leq 0} \max _{1 \leq j \leq n}\left(\left|\theta_{j}(t)\right| / v_{j}\right)$.

Definition 9. System (3) is said to be exponentially stable if there exist two constants $a>0$ and $b>0$ such that

$$
\|\mathbf{x}(t)\| \leq a e^{-b t}\|\mathbf{x}(0)\|, \quad t \geq 0,
$$

where $\|\cdot\|$ is some norm in $\mathbb{R}^{n}$.

The following theorem states a sufficient condition for exponential stability of system (3) with $0<\alpha \leq 1$.

Theorem 10. For system (3) with the degree of $0<\alpha \leq 1$, suppose that Assumptions 5 and 6 hold. If there exists a vector $v \gg \mathbf{0}$ such that

$$
\mathbf{f}(\boldsymbol{v})+\mathbf{g}(\boldsymbol{v}) \ll \mathbf{0},
$$

then system (3) is exponentially stable for any nonnegative initial conditions. In particular, every solution of system (3) satisfies

$$
\|\mathbf{x}(t)\|_{\infty}^{\boldsymbol{v}} \leq e^{\xi d_{\max }}\|\boldsymbol{\theta}\| e^{-\xi t}, \quad t \geq 0,
$$

where $0<\xi<\min _{1 \leq j \leq n} \xi_{j}$ and $\xi_{j}$ is the unique positive solution to

$$
\|\boldsymbol{\theta}\| \xi_{j}+\|\boldsymbol{\theta}\|^{\alpha}\left(\frac{f_{j}(\boldsymbol{v})}{v_{j}}+e^{\xi_{j} d_{\max } \alpha} \frac{g_{j}(\boldsymbol{v})}{v_{j}}\right)=0 .
$$

Proof. Note that (10) has two parameters: the maximum delay $d_{\max }$ and $\xi_{j}$. So, naturally for fixed $d_{\max } \geq 0$, define a set of functions with respect to $\xi$ :

$$
\begin{array}{r}
\varphi_{j}(\xi)=\|\boldsymbol{\theta}\| \xi+\|\boldsymbol{\theta}\|^{\alpha}\left(\frac{f_{j}(\boldsymbol{v})}{v_{j}}+e^{\xi d_{\max } \alpha} \frac{g_{j}(\boldsymbol{v})}{v_{j}}\right), \\
j=1,2, \ldots, n .
\end{array}
$$

It now follows from (10) that

$$
\varphi_{j}\left(\xi_{j}\right)=0 \quad \forall j \in\{1,2, \ldots, n\} .
$$

Furthermore, it can be easily checked that $\varphi_{j}(\xi)$ is strictly monotonically increasing in $\xi>0$, which implies that

$$
\begin{array}{cc}
\varphi_{1}(\xi)<0, & \xi<\xi_{1}, \\
\varphi_{2}(\xi)<0, & \xi<\xi_{2}, \\
\vdots & \\
\varphi_{n}(\xi)<0, & \xi<\xi_{n} .
\end{array}
$$

So if $\xi \in\left(0, \min _{1 \leq j \leq n} \xi_{j}\right)$, we have

$$
\begin{array}{r}
\varphi_{j}(\xi)=\|\boldsymbol{\theta}\| \xi+\|\boldsymbol{\theta}\|^{\alpha}\left(\frac{f_{j}(\boldsymbol{v})}{v_{j}}+e^{\xi d_{\max } \alpha} \frac{g_{j}(\boldsymbol{v})}{v_{j}}\right)<0 \\
\forall j \in\{1,2, \ldots, n\} .
\end{array}
$$

Now, inspired by the method developed in [6], let

$$
\omega_{j}(t)=e^{\xi t} \frac{x_{j}(t)}{v_{j}}-e^{\xi d_{\max }}\|\boldsymbol{\theta}\| .
$$

Then it follows from Lemma 8 that

$$
\begin{aligned}
e^{\xi t} \frac{x_{j}(t)}{v_{j}} \leq e^{\xi d_{\max }} \frac{x_{j}(t)}{v_{j}} & \leq e^{\xi d_{\max }}\|\boldsymbol{\theta}\| \\
\forall t & \in\left[0, d_{\max }\right], j \in\{1,2, \ldots, n\} .
\end{aligned}
$$

It is immediate to see that, for any $j \in\{1,2, \ldots, n\}$,

$$
\omega_{j}(t) \leq 0, \quad 0 \leq t \leq d_{\max } .
$$

In order to prove exponential stability of system (3), we still need to prove that, for all $j$ and all $t \geq d_{\text {max }}, \omega_{j}(t) \leq 0$. Obviously, $\left.\omega_{j}(t)\right|_{t=d_{\max }} \leq 0$ for all $j$. As $\omega_{j}(t)$ is continuous function, we claim that it is also holds for all $t \geq d_{\max }$ and all $j$. By contradiction, suppose that this is not always true. Then there exist an index $s \in\{1,2, \ldots, n\}$ and a time $t^{*} \in$ $\left[d_{\max },+\infty\right)$ such that

$$
\begin{aligned}
\omega_{j}(t) & \leq 0 \quad \forall t \in\left[d_{\max }, t^{*}\right], j \in\{1,2, \ldots, n\}, \\
\omega_{s}\left(t^{*}\right) & =0 \\
\left.D^{+} \omega_{s}(t)\right|_{t=t^{*}} & \geq 0 .
\end{aligned}
$$

From (18) and (19), we have

$$
\begin{aligned}
& x_{j}\left(t^{*}\right) \leq e^{\xi d_{\max }} e^{-\xi t^{*}}\|\boldsymbol{\theta}\| v_{j}, \quad j=1,2, \ldots, n, j \neq s, \\
& x_{s}\left(t^{*}\right)=e^{\xi d_{\max }} e^{-\xi t^{*}}\|\boldsymbol{\theta}\| v_{s} .
\end{aligned}
$$

Then it follows from cooperativity and homogeneity of $\mathbf{f}$ that

$$
f_{s}\left(\mathbf{x}\left(t^{*}\right)\right) \leq\left(e^{\xi d_{\max }} e^{-\xi t^{*}}\right)^{\alpha}\|\boldsymbol{\theta}\|^{\alpha} f_{s}(\boldsymbol{v}) .
$$


Note that $t^{*}-\tau\left(t^{*}\right) \in\left(0, t^{*}\right]$, so we obtain

$$
\mathbf{x}\left(t^{*}-\tau\left(t^{*}\right)\right) \leq e^{\xi d_{\max }} e^{-\xi\left(t^{*}-\tau\left(t^{*}\right)\right)}\|\boldsymbol{\theta}\| \boldsymbol{v} .
$$

Furthermore, as $\mathbf{g}$ is order-preserving and homogeneous, it in turn implies that

$$
g_{s}\left(\mathbf{x}\left(t^{*}-\tau\left(t^{*}\right)\right)\right) \leq\left(e^{\xi d_{\max }} e^{-\xi\left(t^{*}-\tau\left(t^{*}\right)\right)}\right)^{\alpha}\|\boldsymbol{\theta}\|^{\alpha} g_{s}(\boldsymbol{v})
$$

The upper-right Dini derivative of $\omega_{s}(t)$ along the trajectories of system (3) at $t=t^{*}$ is given by

$$
\begin{aligned}
&\left.D^{+} \omega_{s}(t)\right|_{t=t^{*}}=\xi e^{\xi t^{*}} \frac{x_{s}\left(t^{*}\right)}{v_{s}}+e^{\xi t^{*}} \frac{\dot{x}_{s}\left(t^{*}\right)}{v_{s}} \\
&=\xi e^{\xi t^{*}} \frac{x_{s}\left(t^{*}\right)}{v_{s}} \\
& \quad+e^{\xi t^{*}} \frac{f_{s}\left(\mathbf{x}\left(t^{*}\right)\right)+g_{s}\left(\mathbf{x}\left(t^{*}-\tau\left(t^{*}\right)\right)\right)}{v_{s}} \\
& \leq \xi e^{\xi d_{\max }}\|\boldsymbol{\theta}\| \\
& \quad+e^{\xi t^{*}(1-\alpha)+\xi d_{\max } \alpha}\|\boldsymbol{\theta}\|^{\alpha}\left(\frac{f_{s}(\boldsymbol{v})}{v_{s}}+e^{\xi \tau\left(t^{*}\right) \alpha} \frac{g_{s}(\boldsymbol{v})}{v_{s}}\right) \\
& \leq \xi e^{\xi d_{\max }}\|\boldsymbol{\theta}\| \\
&+e^{\xi\left[t^{*}+\alpha\left(d_{\max }-t^{*}\right)\right]}\|\boldsymbol{\theta}\|^{\alpha}\left(\frac{f_{s}(\boldsymbol{v})}{v_{s}}+e^{\xi d_{\max } \alpha} \frac{g_{s}(\boldsymbol{v})}{v_{s}}\right) \\
& \leq \xi e^{\xi d_{\max }}\|\boldsymbol{\theta}\| \\
&+e^{\xi d_{\max }}\|\boldsymbol{\theta}\|^{\alpha}\left(\frac{f_{s}(\boldsymbol{v})}{v_{s}}+e^{\xi d_{\max } \alpha} \frac{g_{s}(\boldsymbol{v})}{v_{s}}\right) \\
& v_{\max }\left.\|\boldsymbol{\theta}\| \xi+\|\boldsymbol{\theta}\|^{\alpha}\left(\frac{f_{s}(\boldsymbol{v})}{v_{s}}+e^{\xi d_{\max } \alpha} \frac{g_{s}(\boldsymbol{v})}{v_{s}}\right)\right) \\
& 0,
\end{aligned}
$$

where we have used (22) and (24) to get the first inequality. Note also that $0 \leq \tau(t) \leq d_{\max }$ and $f_{s}(\boldsymbol{v}) / v_{s}+e^{\xi d_{\max } \alpha} g_{s}(\boldsymbol{v}) / v_{s}<$ 0 , so the second and third inequalities are true, and the last inequality is from (14). Now we arrive at a contradiction with (20). Hence, $\omega_{j}(t) \leq 0$; for all $t \geq 0, j \in\{1,2, \ldots, n\}$, which means that

$$
\|\mathbf{x}(t)\|_{\infty}^{v} \leq e^{\xi d_{\max }}\|\boldsymbol{\theta}\| e^{-\xi t}, \quad t \in[0,+\infty) .
$$

This completes the proof.

Remark 11. Theorem 10 provides an estimate on how the decay rate depends on initial condition with the degree of $0<\alpha \leq 1$, which is fundamental to study the switched positive systems.

Next, consider the corresponding delay-free system:

$$
\dot{\mathbf{x}}(t)=\mathbf{f}(\mathbf{x}(t))+\mathbf{g}(\mathbf{x}(t)) .
$$

In the following, we give a sufficient and necessary condition for the exponential stability of system (27).
Corollary 12. Consider system (27) with $0<\alpha \leq 1$ under Assumption 5; then the following statements are equivalent:

(1) There exists a vector $\boldsymbol{v} \gg \mathbf{0}$ such that $\mathbf{f}(\boldsymbol{v})+\mathbf{g}(\boldsymbol{v}) \ll \mathbf{0}$.

(2) System (27) is exponentially stable and every solution $\mathbf{x}(t)$ satisfies

$$
\|\mathbf{x}(t)\|_{\infty}^{v} \leq\|\mathbf{x}(0)\|_{\infty}^{v} e^{-\gamma t}
$$

where $0<\gamma<\min _{1 \leq j \leq n} \gamma_{j}$ with $\gamma_{j}$ being the unique positive solution to

$$
\|\mathbf{x}(0)\|_{\infty}^{\boldsymbol{v}} \gamma_{j}+\left(\|\mathbf{x}(0)\|_{\infty}^{\boldsymbol{v}}\right)^{\alpha}\left(\frac{f_{j}(\boldsymbol{v})}{v_{j}}+\frac{g_{j}(\boldsymbol{v})}{v_{j}}\right)=0 .
$$

Proof. (1) $\Rightarrow$ (2): according to Theorem 10, system (3) is exponentially stable for all delays satisfying Assumption 6 . Particularly, let $\tau(t)=0$; then $d_{\max }=0$ and $\|\boldsymbol{\theta}\|=\|\mathbf{x}(0)\|_{\infty}^{v}$, which implies that (29) holds.

$(2) \Rightarrow(1)$ : as system (27) is exponentially stable, it is asymptotically stable. Therefore, it follows from [20, Proposition 3.10, Theorem 3.12] that there exists a vector $\boldsymbol{v} \gg \mathbf{0}$ satisfying $\mathbf{f}(\boldsymbol{v})+\mathbf{g}(\boldsymbol{v}) \ll \mathbf{0}$.

Remark 13. From (29), one can verify that

$$
\|\mathbf{x}(0)\|_{\infty}^{\boldsymbol{v}} \gamma+\left(\|\mathbf{x}(0)\|_{\infty}^{v}\right)^{\alpha}\left(\frac{f_{j}(\boldsymbol{v})}{v_{j}}+\frac{g_{j}(\boldsymbol{v})}{v_{j}}\right)<0 .
$$

We now consider two cases.

Case $1\left(\|\mathbf{x}(0)\|_{\infty}^{v} \leq 1\right)$. As $0<\alpha \leq 1$, we can get $\|\mathbf{x}(0)\|_{\infty}^{v} \leq$ $\left(\|\mathbf{x}(0)\|_{\infty}^{v}\right)^{\alpha}$. Hence, it follows from $f_{j}(\boldsymbol{v}) / v_{j}+g_{j}(\boldsymbol{v}) / v_{j}<0$ that

$$
\begin{gathered}
\|\mathbf{x}(0)\|_{\infty}^{\boldsymbol{v}} \gamma+\left(\|\mathbf{x}(0)\|_{\infty}^{v}\right)^{\alpha}\left(\frac{f_{j}(\boldsymbol{v})}{v_{j}}+\frac{g_{j}(\boldsymbol{v})}{v_{j}}\right) \\
\leq\|\mathbf{x}(0)\|_{\infty}^{\boldsymbol{v}}\left(\gamma+\frac{f_{j}(\boldsymbol{v})}{v_{j}}+\frac{g_{j}(\boldsymbol{v})}{v_{j}}\right) .
\end{gathered}
$$

Case $2\left(\|\mathbf{x}(0)\|_{\infty}^{v}>1\right)$. From $0<\alpha \leq 1$, we have $\left(\|\mathbf{x}(0)\|_{\infty}^{v}\right)^{\alpha}>$ 1. Thus

$$
\begin{aligned}
& \|\mathbf{x}(0)\|_{\infty}^{v} \gamma+\left(\|\mathbf{x}(0)\|_{\infty}^{v}\right)^{\alpha}\left(\frac{f_{j}(\boldsymbol{v})}{v_{j}}+\frac{g_{j}(\boldsymbol{v})}{v_{j}}\right) \\
& \leq\|\mathbf{x}(0)\|_{\infty}^{v} \gamma+\frac{f_{j}(\boldsymbol{v})}{v_{j}}+\frac{g_{j}(\boldsymbol{v})}{v_{j}} .
\end{aligned}
$$

Next, let $\gamma_{j}, \gamma_{j}^{\prime}$ be the positive solutions of the following equations:

$$
\begin{array}{r}
\gamma_{j}+\frac{f_{j}(\boldsymbol{v})}{v_{j}}+\frac{g_{j}(\boldsymbol{v})}{v_{j}}=0, \\
\|\mathbf{x}(0)\|_{\infty}^{\boldsymbol{v}} \gamma_{j}^{\prime}+\frac{f_{j}(\boldsymbol{v})}{v_{j}}+\frac{g_{j}(\boldsymbol{v})}{v_{j}}=0 .
\end{array}
$$


If $\gamma \in\left(0, \min _{1 \leq j \leq n}\left\{\gamma_{j}, \gamma_{j}^{\prime}\right\}\right)$, then we have

$$
\begin{array}{r}
\gamma+\frac{f_{j}(\boldsymbol{v})}{v_{j}}+\frac{g_{j}(\boldsymbol{v})}{v_{j}}<0, \\
\|\mathbf{x}(0)\|_{\infty}^{\boldsymbol{v}} \gamma+\frac{f_{j}(\boldsymbol{v})}{v_{j}}+\frac{g_{j}(\boldsymbol{v})}{v_{j}}<0 .
\end{array}
$$

Hence, it is easy to verify that (34) ensures that (30) holds. Meanwhile, from the proof of Theorem 10, (30) is the key factor to prove the exponential stability of system (27).

\subsection{Exponential Stability of Switched Positive Homogeneous} Systems. In the following, we consider the following switched positive nonlinear system:

$$
\dot{\mathbf{x}}(t)=\mathbf{f}_{\boldsymbol{\sigma}(t)}(\mathbf{x}(t))+\mathbf{g}_{\boldsymbol{\sigma}(t)}(\mathbf{x}(t)),
$$

where $\mathbf{x}(t) \in \mathbb{R}^{n}$ is the state vector and $\sigma(t):[0,+\infty) \rightarrow M$ denotes the switching signal. The finite set $M=\{1,2, \ldots, m\}$ is an index set and stands for the collection of subsystems. For all $p \in M, \mathbf{f}_{\mathbf{p}}$ and $\mathbf{g}_{\mathbf{p}}: \mathbb{R}^{n} \rightarrow \mathbb{R}^{n}$ are continuously differentiable on $\mathbb{R}^{n} \backslash\{\mathbf{0}\}$.

Remark 14. It should be pointed that, for every $p \in M$, as $\mathbf{f}_{\mathbf{p}}$ is defined to be cooperative and homogeneous and $\mathbf{g}_{\mathbf{p}}$ is orderpreserving and homogeneous, system (35) is positive under arbitrary switching laws. This implies that, for any initial condition $\mathbf{x}(0) \in \mathbb{R}_{+}^{n}$, the corresponding state trajectory $\mathbf{x}(t) \epsilon$ $\mathbb{R}_{+}^{n}$ for all $t \geq 0$.

Lemma 15 (see [19]). Assume that $\mathbf{x} \in \mathbb{R}^{n}$; then, for any $p, q \in$ $M$,

$$
\|\mathbf{x}\|_{\infty}^{\bar{v}} \leq\|\mathbf{x}\|_{\infty}^{v_{\mathrm{p}}} \leq\|\mathbf{x}\|_{\infty}^{v_{\infty}} \leq \beta\|\mathbf{x}\|_{\infty}^{v_{\mathrm{q}}},
$$

where $\beta=\max _{1 \leq j \leq n}\left(\bar{v}_{j} / \underline{v}_{j}\right)$ with $\bar{v}_{j}=\max _{p \in M} v_{p j}, \underline{v}_{j}=$ $\min _{p \in M} v_{p j}, \overline{\boldsymbol{v}}=\left[\bar{v}_{1}, \bar{v}_{2}, \ldots, \bar{v}_{n}\right]$, and $\underline{\boldsymbol{v}}=\left[\underline{v_{1}}, \underline{v_{2}}, \ldots, \underline{v_{n}}\right]$.

Based on Theorem 10 and Lemma 15, we next establish a sufficient condition for exponential stability of switched system (35) and the symbols that are defined in Lemma 15 continue to be used. In addition, let $Q=\left\{\mathbf{x} \in \mathbb{R}_{+}^{n} \backslash\{\mathbf{0}\} \mid\right.$ $\left.x_{i} \leq a_{i}, a_{i} \in \mathbb{R}, i=1,2, \ldots, n\right\}$, where $a_{i}>0$ is any given constant. Further define

$$
\varepsilon=\sup _{\mathbf{x} \in Q}\|\mathbf{x}\|_{\infty}^{v} .
$$

Theorem 16. For system (35), suppose that, for every $p \in M$, $\mathbf{f}_{\mathbf{p}}, \mathbf{g}_{\mathbf{p}}$ satisfy Assumption 5 with $0<\alpha \leq 1$. If there exists $a$ vector $v_{\mathbf{p}} \gg \mathbf{0}$ such that

$$
\mathbf{f}_{\mathbf{p}}\left(v_{\mathbf{p}}\right)+\mathbf{g}_{\mathbf{p}}\left(v_{\mathbf{p}}\right) \ll \mathbf{0}, \quad \forall p \in M,
$$

then system (35) is exponentially stable for any given set $Q$ under MDT switching signal $\tau$ satisfying

$$
\tau>\frac{\ln \beta}{\lambda},
$$

where $0<\lambda<\min \{\mu, \eta\}$ with $\mu=\min _{p \in M} \min _{1 \leq j \leq n} \lambda_{p j}$ and $\eta=\min _{p \in M} \min _{1 \leq j \leq n} \lambda_{p j}^{*}$ and $\lambda_{p j}$ and $\lambda_{p j}^{*}$ are the solutions to the following equations:

$$
\begin{gathered}
\lambda_{p j}+\frac{f_{p j}\left(\boldsymbol{v}_{\mathbf{p}}\right)}{v_{p j}}+\frac{g_{p j}\left(\boldsymbol{v}_{\mathbf{p}}\right)}{v_{p j}}=0, \quad p \in M, \\
\varepsilon \lambda_{p j}^{*}+\frac{f_{p j}\left(\boldsymbol{v}_{\mathbf{p}}\right)}{v_{p j}}+\frac{g_{p j}\left(\boldsymbol{v}_{\mathbf{p}}\right)}{v_{p j}}=0, \quad p \in M .
\end{gathered}
$$

Proof. Consider a switching sequence

$$
0=t_{0}<t_{1}<t_{2}<\cdots<t_{k}<t_{k+1}<\cdots .
$$

From (39), we have $\Delta t_{k}=t_{k+1}-t_{k} \geq \tau>\ln \beta / \lambda, k \in \mathbb{N}_{0}$.

The proof now proceeds in two steps.

Step 1. First, for any $\mathbf{x}(0) \in Q$, we show that

$$
\begin{aligned}
&\left\|\mathbf{x}\left(t_{k}\right)\right\|_{\infty}^{\boldsymbol{v}_{\left(t_{k-1}\right)}} \leq\left\|\mathbf{x}\left(t_{k-1}\right)\right\|_{\infty}^{\boldsymbol{v}_{\boldsymbol{\sigma}\left(t_{k-1}\right)}} e^{-\lambda\left(t_{k}-t_{k-1}\right)}, \quad k \in \mathbb{N} \\
&\left\|\mathbf{x}\left(t_{k}\right)\right\|_{\infty}^{\boldsymbol{v}_{\boldsymbol{\sigma}\left(t_{k}\right)}} \leq\|\mathbf{x}(0)\|_{\infty}^{\boldsymbol{v}_{(0)}}, \quad k \in \mathbb{N} .
\end{aligned}
$$

Note that, for every $p \in M$, there exists $\boldsymbol{v}_{\mathbf{p}} \gg \mathbf{0}$ such that $\mathbf{f}_{p}\left(\boldsymbol{v}_{p}\right)+\mathbf{g}_{p}\left(\boldsymbol{v}_{p}\right) \ll \mathbf{0}$, so, from Corollary 12 , for the first interval $\left[t_{0}, t_{1}\right)$, we have

$$
\left\|\mathbf{x}\left(t_{1}\right)\right\|_{\infty}^{v_{\sigma(0)}} \leq\|\mathbf{x}(0)\|_{\infty}^{v_{\sigma(0)}} e^{-\lambda t_{1}} \leq \frac{1}{\beta}\|\mathbf{x}(0)\|_{\infty}^{v_{\sigma(0)}}
$$

where we have used $\Delta t_{1}=t_{1} \geq \tau>\ln \beta / \lambda$ to get the second inequality. Furthermore, we obtain

$$
\begin{aligned}
\left\|\mathbf{x}\left(t_{1}\right)\right\|_{\infty}^{\boldsymbol{v}_{\boldsymbol{\sigma}\left(t_{1}\right)}} & \leq \beta\left\|\mathbf{x}\left(t_{1}\right)\right\|_{\infty}^{\boldsymbol{v}_{\boldsymbol{\sigma}(0)}} \leq \beta \frac{1}{\beta}\|\mathbf{x}(0)\|_{\infty}^{\boldsymbol{v}_{\boldsymbol{\sigma}(0)}} \\
& =\|\mathbf{x}(0)\|_{\infty}^{\boldsymbol{v}_{\boldsymbol{\sigma}(0)}} .
\end{aligned}
$$

Next, consider the second interval $\left[t_{1}, t_{2}\right)$. It follows from Corollary 12 that

$$
\left\|\mathbf{x}\left(t_{2}\right)\right\|_{\infty}^{\boldsymbol{v}_{\boldsymbol{\sigma}\left(t_{1}\right)}} \leq\left\|\mathbf{x}\left(t_{1}\right)\right\|_{\infty}^{\boldsymbol{v}_{\boldsymbol{\sigma}\left(t_{1}\right)}} e^{-\lambda_{1}\left(t_{2}-t_{1}\right)} .
$$

From Remark 13, we have $0<\lambda_{1}<\min _{1 \leq j \leq n}\left\{\bar{\lambda}_{j}, \bar{\lambda}_{j}^{\prime}\right\}$, where $\bar{\lambda}_{j}$ and $\bar{\lambda}_{j}^{\prime}$ are the positive solutions of the following equations:

$$
\begin{array}{r}
\bar{\lambda}_{j}+\frac{f_{v_{\sigma\left(t_{1}\right) j}}\left(\boldsymbol{v}_{\boldsymbol{\sigma}\left(t_{1}\right)}\right)}{v_{\sigma\left(t_{1}\right) j}}+\frac{g_{v_{\sigma\left(t_{1}\right) j}}\left(\boldsymbol{v}_{\boldsymbol{\sigma}\left(t_{1}\right)}\right)}{v_{\sigma\left(t_{1}\right) j}}=0, \\
\left\|\mathbf{x}\left(t_{1}\right)\right\|_{\infty}^{\boldsymbol{v}_{\boldsymbol{\sigma}\left(t_{1}\right)}} \bar{\lambda}_{j}^{\prime}+\frac{f_{v_{\sigma\left(t_{1}\right) j}}\left(\boldsymbol{v}_{\sigma\left(t_{1}\right)}\right)}{v_{\sigma\left(t_{1}\right) j}}+\frac{g_{v_{\sigma\left(t_{1}\right) j}}\left(\boldsymbol{v}_{\sigma\left(t_{1}\right)}\right)}{v_{\sigma\left(t_{1}\right) j}}=0 .
\end{array}
$$


Observe (40) and (47). For $p \in M$, let

$$
\begin{aligned}
S_{p} & =\left\{\lambda_{p j}: \lambda_{p j}+\frac{f_{p j}\left(\boldsymbol{v}_{\mathbf{p}}\right)}{v_{p j}}+\frac{g_{p j}\left(\boldsymbol{v}_{\mathbf{p}}\right)}{v_{p j}}=0, j\right. \\
& =1,2, \ldots, n\}, \\
\bar{S} & =\left\{\bar{\lambda}_{j}: \bar{\lambda}_{j}+\frac{f_{v_{\sigma\left(t_{1}\right) j}}\left(\boldsymbol{v}_{\boldsymbol{\sigma}\left(t_{1}\right)}\right)}{v_{\sigma\left(t_{1}\right) j}}+\frac{g_{v_{\sigma\left(t_{1}\right) j}}\left(\boldsymbol{v}_{\boldsymbol{\sigma}\left(t_{1}\right)}\right)}{v_{\sigma\left(t_{1}\right) j}}\right. \\
& =0, j=1,2, \ldots, n\} .
\end{aligned}
$$

It is easy to verify that $\bar{S} \subset \bigcup_{p \in M} S_{p}$; thus

$$
\min _{p \in M} \min _{\lambda_{p j} \in S_{p}} \lambda_{p j} \leq \min _{\bar{\lambda}_{j} \in \bar{S}} \bar{\lambda}_{j} .
$$

That is,

$$
\mu \leq \min _{1 \leq j \leq n} \bar{\lambda}_{j}
$$

On the other hand, from (45), we have $\left\|\mathbf{x}\left(t_{1}\right)\right\|_{\infty}^{v_{\sigma\left(t_{1}\right)}} \leq$ $\|\mathbf{x}(0)\|_{\infty}^{\boldsymbol{v}_{\sigma(0)}}$. Furthermore, from the definition of $\varepsilon$, we can get $\left\|\mathbf{x}\left(t_{1}\right)\right\|_{\infty}^{\boldsymbol{v}_{\sigma\left(t_{1}\right)}} \leq \varepsilon$. Hence, comparing (41) and (48), one can check that

$$
\eta \leq \min _{1 \leq j \leq n} \bar{\lambda}_{j}^{\prime}
$$

This together with (51) implies that

$$
\min \{\mu, \eta\} \leq \min _{1 \leq j \leq n}\left\{\bar{\lambda}_{j}, \bar{\lambda}_{j}^{\prime}\right\}
$$

So if for any $\lambda_{1} \in\left(0, \min _{1 \leq j \leq n}\left\{\bar{\lambda}_{j}, \bar{\lambda}_{j}^{\prime}\right\}\right)(46)$ holds, then

$$
\left\|\mathbf{x}\left(t_{2}\right)\right\|_{\infty}^{\boldsymbol{v}_{\boldsymbol{\sigma}\left(t_{1}\right)}} \leq\left\|\mathbf{x}\left(t_{1}\right)\right\|_{\infty}^{\boldsymbol{v}_{\boldsymbol{\sigma}\left(t_{1}\right)}} e^{-\lambda\left(t_{2}-t_{1}\right)}
$$

where $\lambda \in(0, \min \{\mu, \eta\})$. Now it follows from Lemma 15 that

$$
\begin{aligned}
\left\|\mathbf{x}\left(t_{2}\right)\right\|_{\infty}^{v_{\left(t_{2}\right)}} & \leq \beta\left\|\mathbf{x}\left(t_{2}\right)\right\|_{\infty}^{v_{\sigma\left(t_{1}\right)}} \leq \beta \frac{1}{\beta}\left\|\mathbf{x}\left(t_{1}\right)\right\|_{\infty}^{\boldsymbol{v}_{\sigma\left(t_{1}\right)}} \\
& \leq\|\mathbf{x}(0)\|_{\infty}^{\boldsymbol{v}_{\sigma(0)}}
\end{aligned}
$$

where we have used (39) and (54) to get the second inequality. From (44), (45), (54), and (55), we conclude that (43) hold for $k=1,2$.

By induction, we assume that (43) hold for a given $k(k \geq$ 3). Next we prove they are true for $k+1$. Consider the interval $\left[t_{k}, t_{k+1}\right)$. It follows from Corollary 12 that

$$
\left\|\mathbf{x}\left(t_{k+1}\right)\right\|_{\infty}^{\boldsymbol{v}_{\boldsymbol{\sigma}\left(t_{k}\right)}} \leq\left\|\mathbf{x}\left(t_{k}\right)\right\|_{\infty}^{\boldsymbol{v}_{\boldsymbol{\sigma}\left(t_{k}\right)}} e^{-\lambda_{k}\left(t_{k+1}-t_{k}\right)},
$$

where $0<\lambda_{k}<\min _{1 \leq j \leq n}\left\{\tilde{\lambda}_{j}, \tilde{\lambda}_{j}^{\prime}\right\}$ with $\tilde{\lambda}_{j}$ and $\tilde{\lambda}_{j}^{\prime}$ satisfying

$$
\begin{array}{r}
\tilde{\lambda}_{j}+\frac{f_{v_{\sigma\left(t_{k}\right) j}}\left(\boldsymbol{v}_{\boldsymbol{\sigma}\left(t_{k}\right)}\right)}{v_{\sigma\left(t_{k}\right) j}}+\frac{g_{v_{\sigma\left(t_{k}\right) j}}\left(\boldsymbol{v}_{\boldsymbol{\sigma}\left(t_{k}\right)}\right)}{v_{\sigma\left(t_{k}\right) j}}=0, \\
\left\|\mathbf{x}\left(t_{k}\right)\right\|_{\infty}^{v_{\sigma\left(t_{k}\right)}} \tilde{\lambda}_{j}^{\prime}+\frac{f_{v_{\sigma\left(t_{k}\right) j}}\left(\boldsymbol{v}_{\sigma\left(t_{k}\right)}\right)}{v_{\sigma\left(t_{k}\right) j}}+\frac{g_{v_{\sigma\left(t_{k}\right) j}}\left(\boldsymbol{v}_{\sigma\left(t_{k}\right)}\right)}{v_{\sigma\left(t_{k}\right) j}}=0 .
\end{array}
$$

Then we can prove

$$
\begin{aligned}
\left\|\mathbf{x}\left(t_{k+1}\right)\right\|_{\infty}^{\boldsymbol{\sigma}_{\left(t_{k}\right)}} & \leq\left\|\mathbf{x}\left(t_{k}\right)\right\|_{\infty}^{\boldsymbol{v}_{\boldsymbol{\sigma}\left(t_{k}\right)}} e^{-\lambda\left(t_{k+1}-t_{k}\right)}, \\
\left\|\mathbf{x}\left(t_{k+1}\right)\right\|_{\infty}^{\boldsymbol{v}_{\boldsymbol{\sigma}\left(t_{k+1}\right)}} & \leq\|\mathbf{x}(0)\|_{\infty}^{\boldsymbol{v}_{(0)}} .
\end{aligned}
$$

The rest of the proof is similar to the one for the case of $k=2$ and thus is omitted.

According to the previous arguments, we conclude that (43) hold for each $k \in \mathbb{N}$, which implies that for all subsystems we find the common exponential decay rate $\lambda$. In addition, We also prove that $\left\|\mathbf{x}\left(t_{k}\right)\right\|_{\infty}^{\boldsymbol{v}_{\boldsymbol{\sigma}\left(t_{k}\right)}} \leq\|\mathbf{x}(0)\|_{\infty}^{\boldsymbol{v}_{\boldsymbol{\sigma}(0)}}$ under minimum dwell time switching, where $\mathbf{x}(k)$ is the initial condition of the $(k+1)$ th subsystem.

Step 2. We prove that system (35) is exponentially stable. For any $t \in[0,+\infty)$, assume that $t \in\left[t_{k}, t_{k+1}\right)$ on which $\sigma(t)=p$. Then, from Step 1, we have

$$
\|\mathbf{x}(t)\|_{\infty}^{v_{p}} \leq\left\|\mathbf{x}\left(t_{k}\right)\right\|_{\infty}^{v_{\mathbf{p}}} e^{-\lambda\left(t-t_{k}\right)} .
$$

Moreover, applying Lemma 15, we can get

$$
\begin{gathered}
\|\mathbf{x}(t)\|_{\infty}^{v_{\mathbf{p}}} \leq\left\|\mathbf{x}\left(t_{k}\right)\right\|_{\infty}^{v_{\mathbf{p}}} e^{-\lambda\left(t-t_{k}\right)} \\
\leq \beta\left\|\mathbf{x}\left(t_{k}\right)\right\|_{\infty}^{v_{\boldsymbol{\sigma}\left(t_{k-1}\right)}} e^{-\lambda\left(t-t_{k}\right)} \\
\leq \beta\left\|\mathbf{x}\left(t_{k-1}\right)\right\|_{\infty}^{v_{\sigma\left(t_{k-1}\right)}} e^{-\lambda\left(t-t_{k-1}\right)} \\
\vdots \\
\leq \beta^{t / \tau}\|\mathbf{x}(0)\|_{\infty}^{v_{\sigma(0)}} e^{-\lambda t}
\end{gathered}
$$

where we have used the MDT to obtain the last inequality. On the other hand, note that $\|\mathbf{x}(t)\|_{\infty}^{\bar{v}} \leq\|\mathbf{x}(t)\|_{\infty}^{v_{\mathrm{p}}}$. Therefore

$$
\begin{aligned}
\|\mathbf{x}(t)\|_{\infty}^{\bar{v}} & \leq \beta^{t / \tau}\|\mathbf{x}(0)\|_{\infty}^{v_{\sigma(0)}} e^{-\lambda t} \\
& =e^{(t / \tau) \ln \beta}\|\mathbf{x}(0)\|_{\infty}^{v_{\sigma(0)}} e^{-\lambda t} \\
& =e^{-(\lambda-\ln \beta / \tau) t}\|\mathbf{x}(0)\|_{\infty}^{v_{\sigma(0)}} \\
& \leq \beta\|\mathbf{x}(0)\|_{\infty}^{\bar{v}} e^{-(\lambda-\ln \beta / \tau) t} .
\end{aligned}
$$


In summary, system (35) is exponentially stable for any given set $Q$ under MDT switching.

This is the end of the proof.

\section{Numerical Simulation}

Consider the switched nonlinear positive system consisting of two subsystems given by

$$
\begin{aligned}
& \Gamma_{1}: \dot{\mathbf{x}}(t)=\mathbf{f}_{\mathbf{1}}(\mathbf{x}(t))+\mathbf{g}_{\mathbf{1}}(\mathbf{x}(t)), \\
& \Gamma_{2}: \dot{\mathbf{x}}(t)=\mathbf{f}_{\mathbf{2}}(\mathbf{x}(t))+\mathbf{g}_{\mathbf{2}}(\mathbf{x}(t)),
\end{aligned}
$$

where

$$
\begin{aligned}
& \mathbf{f}_{\mathbf{1}}(\mathbf{x}(t)) \\
& =\left[\begin{array}{l}
\left(x_{1}(t)+2 x_{2}(t)\right)^{1 / 2}-\left(4 x_{1}(t)+x_{2}(t)\right)^{1 / 2} \\
\left(2 x_{1}(t)+x_{2}(t)\right)^{1 / 2}-\left(x_{1}(t)+4 x_{2}(t)\right)^{1 / 2}
\end{array}\right], \\
& \mathbf{g}_{\mathbf{1}}(\mathbf{x}(t))=\left[\begin{array}{l}
\frac{1}{6}\left(x_{1}(t)\right)^{1 / 2} \\
\frac{1}{8}\left(x_{2}(t)\right)^{1 / 2}
\end{array}\right], \\
& \mathbf{f}_{2}(\mathbf{x}(t)) \\
& =\left[\begin{array}{l}
\left(x_{1}(t)+x_{2}(t)\right)^{1 / 2}-\left(3 x_{1}(t)+x_{2}(t)\right)^{1 / 2} \\
\left(x_{1}(t)+x_{2}(t)\right)^{1 / 2}-\left(x_{1}(t)+3 x_{2}(t)\right)^{1 / 2}
\end{array}\right], \\
& \mathbf{g}_{2}(\mathbf{x}(t))=\left[\begin{array}{c}
\frac{1}{7}\left(x_{1}(t)+x_{2}(t)\right)^{1 / 2} \\
\frac{1}{10}\left(2 x_{1}(t)+x_{2}(t)\right)^{1 / 2}
\end{array}\right] .
\end{aligned}
$$

It is easy to verify that $f_{1}, f_{2}, g_{1}$, and $g_{2}$ satisfy the conditions of Theorem 16 with homogeneous degree of $\alpha=1 / 2$ and

$$
\begin{aligned}
& \mathbf{f}_{\mathbf{1}}(10,8)+\mathbf{g}_{\mathbf{1}}(10,8)=[-1.3021,-0.8357]^{T} \ll \mathbf{0}, \\
& \mathbf{f}_{\mathbf{2}}(8,10)+\mathbf{g}_{\mathbf{2}}(8,10)=[-0.9822,-1.4119]^{T} \ll \mathbf{0} .
\end{aligned}
$$

Let $Q=\left\{x \in \mathbb{R}_{+}^{n} \mid x_{i} \leq 8, i=1,2\right\}$; then $\varepsilon=1$. It follows from Theorem 16 that the corresponding switched nonlinear system is exponentially stable under minimum dwell time $\tau$ > 2.2314. Figure 1 shows the actual rate and the upper bound which agree with the implication of Theorem 16.

\section{Conclusion}

In this paper, we derive a sufficient condition for the exponential stability of switched positive homogeneous systems with degree of $0<\alpha \leq 1$. Some results in literature [19] are extended. In addition, a sufficient condition is presented for the exponential stability of the unswitched positive systems with time-varying delays. A numerical example is given to demonstrate the main result. While the stability problem of such system with delays is the subject of ongoing work, we hope to report the result of which in the future.

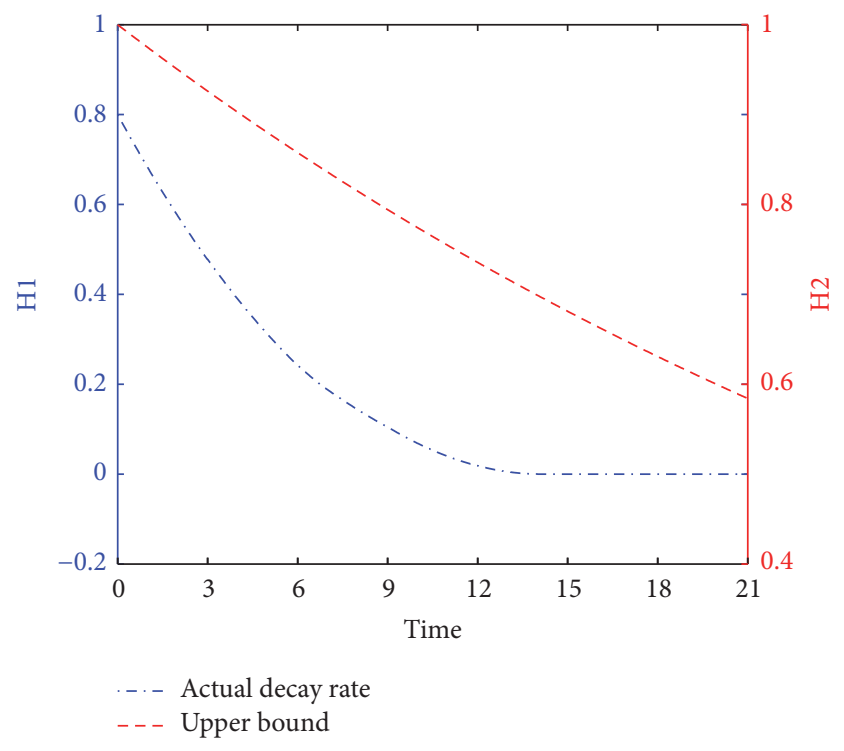

FIGURE 1: The simulation results of the actual decay rate $\|\mathbf{x}(t)\|_{\infty}^{\bar{v}}$ and the guaranteed upper bound we calculated with the initial condition $\mathbf{x}(0)=[7,8]^{T}$ and the MDT $\tau=3$.

\section{Conflicts of Interest}

The authors declare that they have no conflicts of interest.

\section{Acknowledgments}

This work was supported by the Key Program of National Natural Science Foundation of China (no. 61533011) and the National Natural Science Foundation of China (no. 61473133).

\section{References}

[1] H. L. Smith, Monotone Dynamical Systems: An Introduction to the Theory of Competitive and Cooperatiive Systems, vol. 41 of Mathematical Surveys and Monographs, American Mathematical Society, Providence, RI, USA, 1995.

[2] F. Viel, F. Jadot, and G. Bastin, "Global stabilization of exothermic chemical reactors under input constraints," Automatica, vol. 33, no. 8, pp. 1437-1448, 1997.

[3] M. W. Hirsch, "Systems of differential equations that are competitive or cooperative. II. Convergence almost everywhere," SIAM Journal on Mathematical Analysis, vol. 16, no. 3, pp. 423439, 1985.

[4] W. M. Haddad and V. Chellaboina, "Stability theory for nonnegative and compartmental dynamical systems with time delay," Systems \& Control Letters, vol. 51, no. 5, pp. 355-361, 2004.

[5] S. Zhu, Z. Li, and C. Zhang, "Exponential stability analysis for positive systems with delays," IET Control Theory \& Applications, vol. 6, no. 6, pp. 761-767, 2012.

[6] H. R. Feyzmahdavian, T. Charalambous, and M. Johansson, "Exponential stability of homogeneous positive systems of degree one with time-varying delays," Institute of Electrical and Electronics Engineers Transactions on Automatic Control, vol. 59, no. 6, pp. 1594-1599, 2014. 
[7] H. R. Feyzmahdavian, T. Charalambous, and M. Johansson, "Asymptotic stability and decay rates of homogeneous positive systems with bounded and unbounded delays," SIAM Journal on Control and Optimization, vol. 52, no. 4, pp. 2623-2650, 2014.

[8] O. Mason and M. Verwoerd, "Observations on the stability properties of cooperative systems," Systems \& Control Letters, vol. 58, no. 6, pp. 461-467, 2009.

[9] V.S. Bokharaie, O. Mason, and F. Wirth, "Stability and positivity of equilibria for subhomogeneous cooperative systems," Nonlinear Analysis. Theory, Methods \& Applications, vol. 74, no. 17, pp. 6416-6426, 2011.

[10] R. A. Decarlo, M. S. Branicky, S. Pettersson, and B. Lennartson, "Perspectives and results on the stability and stabilizability of hybrid systems," Proceedings of the IEEE, vol. 88, no. 7, pp. 10691082, 2000.

[11] M. S. Alwan and X. Liu, "On stability of linear and weakly nonlinear switched systems with time delay," Mathematical and Computer Modelling, vol. 48, no. 7-8, pp. 1150-1157, 2008.

[12] M. S. Branicky, "Multiple Lyapunov functions and other analysis tools for switched and hybrid systems," Institute of Electrical and Electronics Engineers Transactions on Automatic Control, vol. 43, no. 4, pp. 475-482, 1998.

[13] V. T. Minh, "Stability for switched dynamic hybrid systems," Mathematical and Computer Modelling, vol. 57, no. 1-2, pp. 7883, 2013.

[14] X. Ding, L. Shu, and X. Liu, "On linear copositive Lyapunov functions for switched positive systems," Journal of The Franklin Institute, vol. 348, no. 8, pp. 2099-2107, 2011.

[15] E. Fornasini and M. E. Valcher, "Linear copositive Lyapunov functions for continuous-time positive switched systems," Institute of Electrical and Electronics Engineers Transactions on Automatic Control, vol. 55, no. 8, pp. 1933-1937, 2010.

[16] L. Gurvits, R. Shorten, and O. Mason, "On the stability of switched positive linear systems," Institute of Electrical and Electronics Engineers Transactions on Automatic Control, vol. 52, no. 6, pp. 1099-1103, 2007.

[17] O. Mason and R. Shorten, "On linear copositive Lyapunov functions and the stability of switched positive linear systems," Institute of Electrical and Electronics Engineers Transactions on Automatic Control, vol. 52, no. 7, pp. 1346-1349, 2007.

[18] Y. Sun and $\mathrm{Z}$. Wu, "On the existence of linear copositive Lyapunov functions for 3-dimensional switched positive linear systems," Journal of The Franklin Institute, vol. 350, no. 6, pp. 1379-1387, 2013.

[19] J.-G. Dong, "Stability of switched positive nonlinear systems," International Journal of Robust and Nonlinear Control, vol. 26, no. 14, pp. 3118-3129, 2016.

[20] B. S. Rüffer, C. M. Kellett, and S. R. Weller, "Connection between cooperative positive systems and integral input-to-state stability of large-scale systems," Automatica, vol. 46, no. 6, pp. 1019-1027, 2010. 


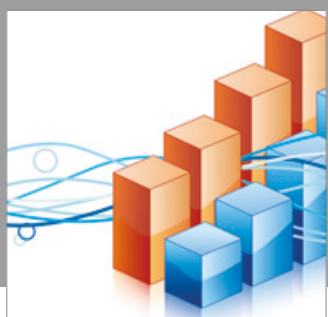

Advances in

Operations Research

vatersals

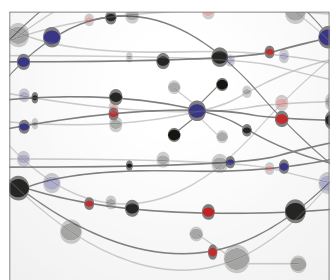

\section{The Scientific} World Journal
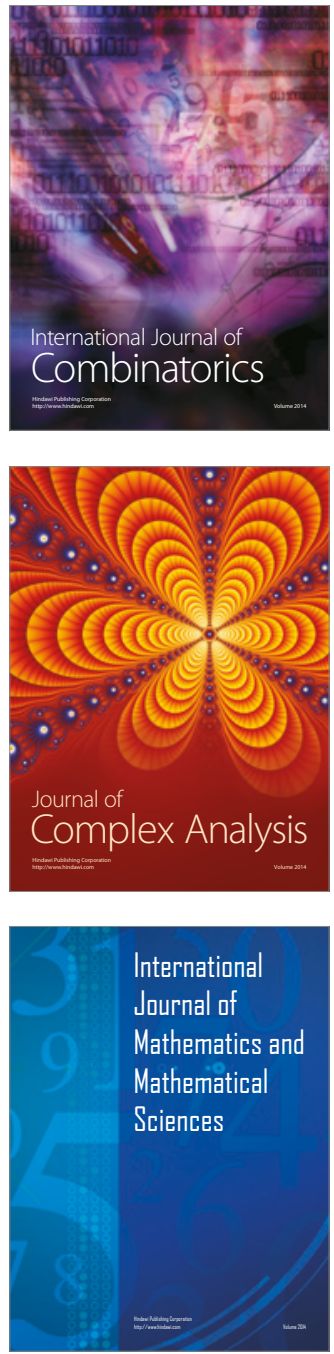
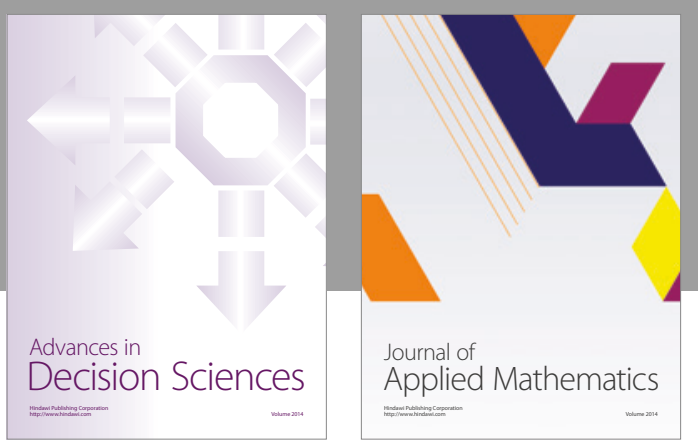

Algebra

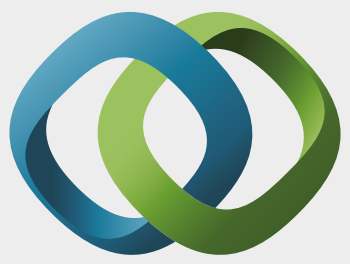

\section{Hindawi}

Submit your manuscripts at

https://www.hindawi.com
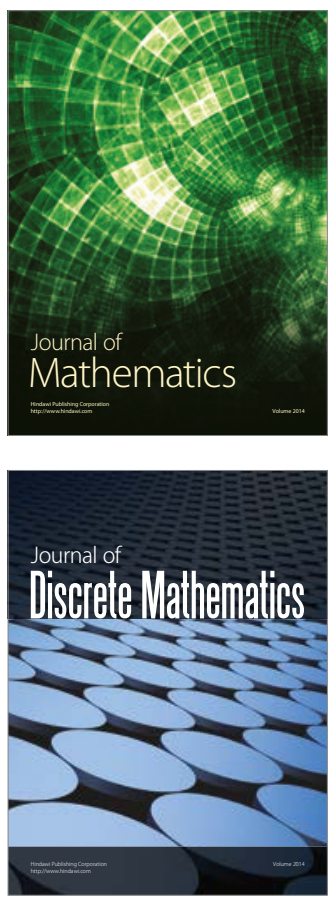

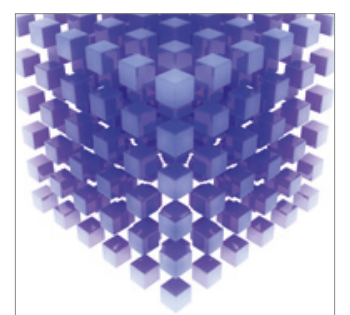

Mathematical Problems in Engineering
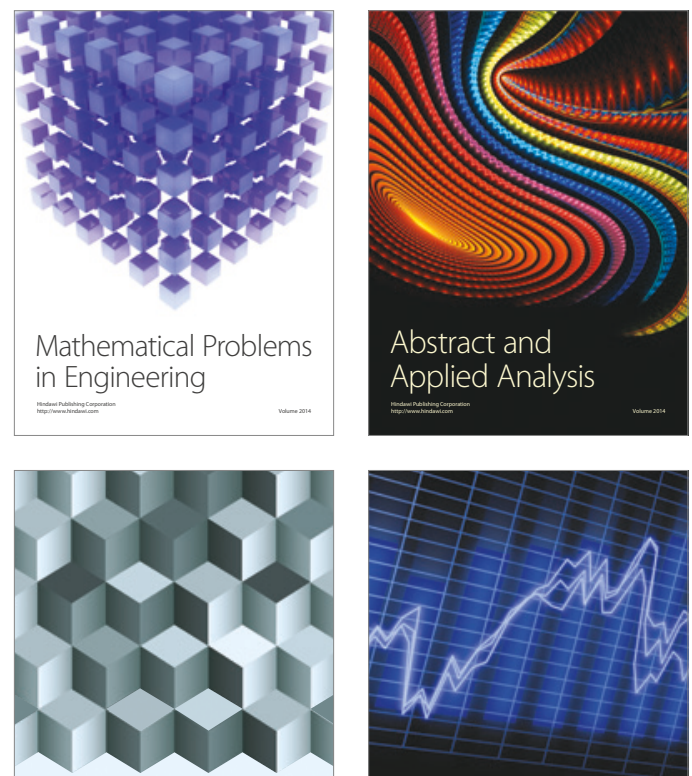

Journal of

Function Spaces

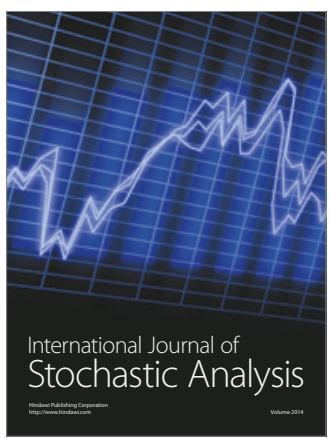

Probability and Statistics
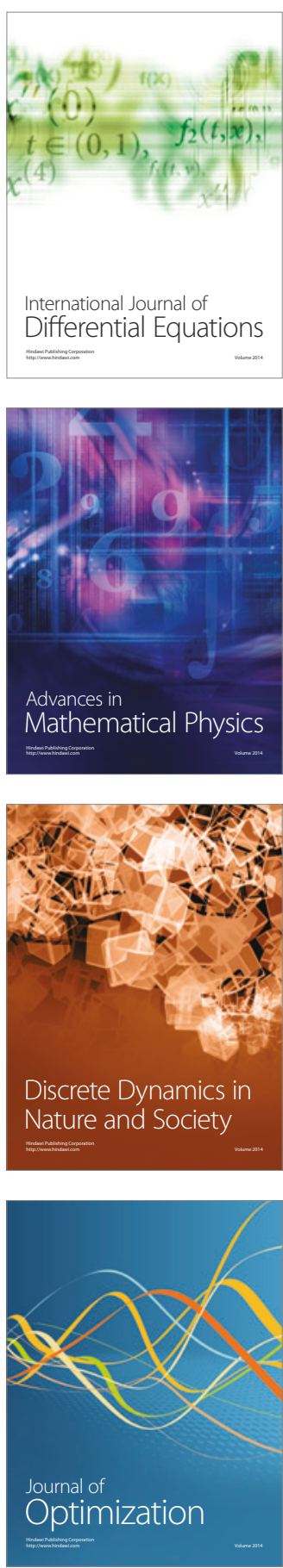